\title{
Erosion et transport solide : ampleur et enjeux
}

\author{
— Erosion and sediment transport : width and stakes -
}

\author{
par Sylvain Ouillon \\ Laboratoire de Sondages Electromagnétiques de l'Environnement Terrestre (LSEET) \\ Université de Toulon et du Var
}

\section{L'étude de l'érosion et du transport solide n'est plus réservée aux besoins du génie civil mais est utilisée aussi pour les besoins de l'agriculture, de l'environnement, des transports et de l'aménagement du territoire.}

\section{L'EAU EN ZONE CONTINENTALE}

Les réserves mondiales en eau sont estimées à un peu plus de 1400 millions de $\mathrm{km}^{3}\left(1 \mathrm{~km}^{3}=10^{9} \mathrm{t}\right)$. La connaissance de la répartition de l'eau et de ses flux est l'objet de l'étude globale du cycle de l'eau (fig. 1). La différence qui apparaît entre les précipitations sur les terres émergées et l'évaporation continentale (évaporation des eaux de surface + évapotranspiration) explique les flux des continents vers les océans, et donc la formation des cours d'eau, dont les principales sources d'alimentation sont les précipitations (pluie, neige), les glaciers et les nappes phréatiques. Le débit global concerné se situe, si l'on tient compte des différentes estimations, entre 35000 et $40000 \mathrm{~km}^{3} / \mathrm{an}\left(38830 \mathrm{~km}^{3} / \mathrm{an}\right.$ selon L'vovich, 1973). La nature des sols, le couvert végétal, le climat, la circulation atmosphérique sont parmi les facteurs qui déterminent, sur chaque zone, les précipitations et l'évaporation. Les disparités géographiques et climatiques ont donc une forte empreinte sur les disparités hydrologiques. Le tableau 1 présente l'estimation des flux pour les princi-

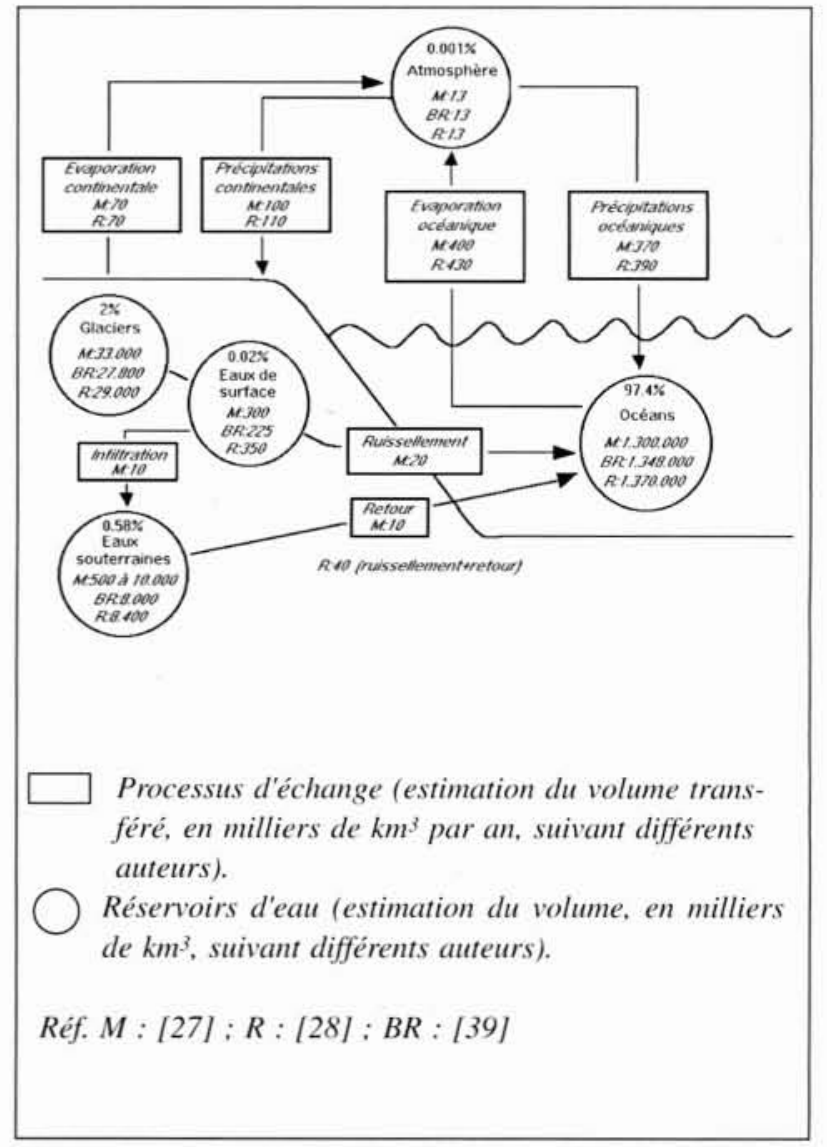

1. Volume des grands réservoirs d'eau de la terre et flux annuels entre les réservoirs.

Although resulting from natural processes that are noticeable by everyone, erosion and sediment transport are shown as scientific topics from only a few decades. Firstly initiated from civil engineering purpose, the need for research and studies is now motivated by queries in agriculture, hygiene, environment quality, transport and regional planning. Within the scope of this paper, the ecological, social and economical stakes related to these questions are collected to keep in prospect research and studies. This paper gathers important assessments relative to erosion and sediment transport. Although we tried to make it as complete as possible, this synthesis is not exhaustive. It deals with erosion and sediment transport ranges and stakes, and it does not tackle neither methods nor tools. 
Tableau 1. - Flux d'eau globaux pour les grands continents (d'après [28]).

\begin{tabular}{|l|c|c|c|cc|}
\hline \multicolumn{1}{|c|}{ Continents } & Surface & Précipitations & Evaporation & \multicolumn{2}{c|}{$\begin{array}{c}\text { Ruissellement } \\
\text { global }\end{array}$} \\
& $\left(10^{6} \mathrm{~km}^{2}\right)$ & $\left(10^{3} \mathrm{~km}^{3} / \mathrm{an}\right)$ & $\left(10^{3} \mathrm{~km}^{3} / \mathrm{an}\right)$ & $\begin{array}{c}\text { au } \\
\left(10^{3} \mathrm{~km}^{3} / \mathrm{an}\right)\end{array}$ \\
\hline Asie & 44,0 & 31 & 18,5 & 12,5 & 0,28 \\
Afrique & 30,2 & 21 & 17 & 4 & 0,13 \\
Amérique du Nord & 23,5 & 15,5 & 9,5 & 6 & 0,25 \\
Amérique du Sud & 18,6 & 27,5 & 16,5 & 3 & 0,59 \\
Europe & 10,0 & 7 & 4 & 3.5 & 0,30 \\
Australie & 7,7 & 7 & 4,5 & 2,5 & 0,32 \\
\hline
\end{tabular}

paux continents. Sur la valeur globale de l'écoulement moyen annuel dans les bassins fluviaux du monde, seuls $14000 \mathrm{~km}^{3}$ environ correspondent à des volumes stables d'écoulement et peuvent servir de base pour une gestion rationnelle des ressources en eau de la biosphère.

Hormis le transit des masses d'eau des continents vers les océans, en dehors de toute activité humaine, les milieux aquatiques ont des rôles majeurs dans la dynamique de la planète, en recevant, transportant ou assimilant les matières arrachées aux bassins versants et en abritant une faune et une flore très variées au sein de multiples écosystèmes. Par ailleurs, l'homme utilise également les capacités propres de l'eau et des milieux aquatiques à ses fins. Plusieurs classifications des usages de l'eau existent, mettant en relief les origines, causes ou conséquences de ses pratiques [1]. A chacune de ces utilisations de l'eau sont associés des problèmes posés par le transport de particules, d'origine naturelle ou anthropique.

\section{II $\square$ ÉROSION DES SOLS}

\subsection{Processus et origines}

Le couvert végétal, lorsqu'il existe, protège le sol en retenant l'eau de pluie, lui donnant le temps de percoler vers les réservoirs souterrains pour aller ensuite, graduellement, grossir les cours d'eau. Sur un terrain en pente, lorsqu'il n'y a pas ou plus de couvert végétal, l'eau de pluie ruisselle au lieu d'être retenue et de s'infiltrer. Cela a deux conséquences primordiales : d'une part, les crues y sont potentiellement plus fortes que sur un sol couvert, d'autre part, l'eau arrache et entraîne en plus grande quantité des particules du sol, provoquant alors une importante érosion. La figure 2 illustre cet aspect, présentant l'efficacité érosive des bioclimats calculée à partir de la turbidité des cours d'eau, quantifiant ainsi le rôle protecteur de la végétation vis-à-vis de l'érosion des sols [2]. L'eau n'est pas le seul vecteur de particules. Sur un sol non protégé, le vent déplace ou agrandit les déserts (érosion éolienne). Dans certains bassins versants, dont notamment celui du Huang Ho (fleuve jaune), l'érosion éolienne est prépondérante. Les particules de loess, arrachées par le vent, sont ensuite lessivées par les précipitations.
L'érosion se produit donc essentiellement sur des zones naturellement arides et semi-arides, mais également sur toute zone sur laquelle les activités humaines ont conduit à faire disparaître le couvert végétal, du fait d'une gestion inappropriée des terres cultivées (suppression de jachère), d'un surpâturage ou de la destruction intentionnelle de la végétation (déboisement, feu), généralement motivés par la poussée démographique.

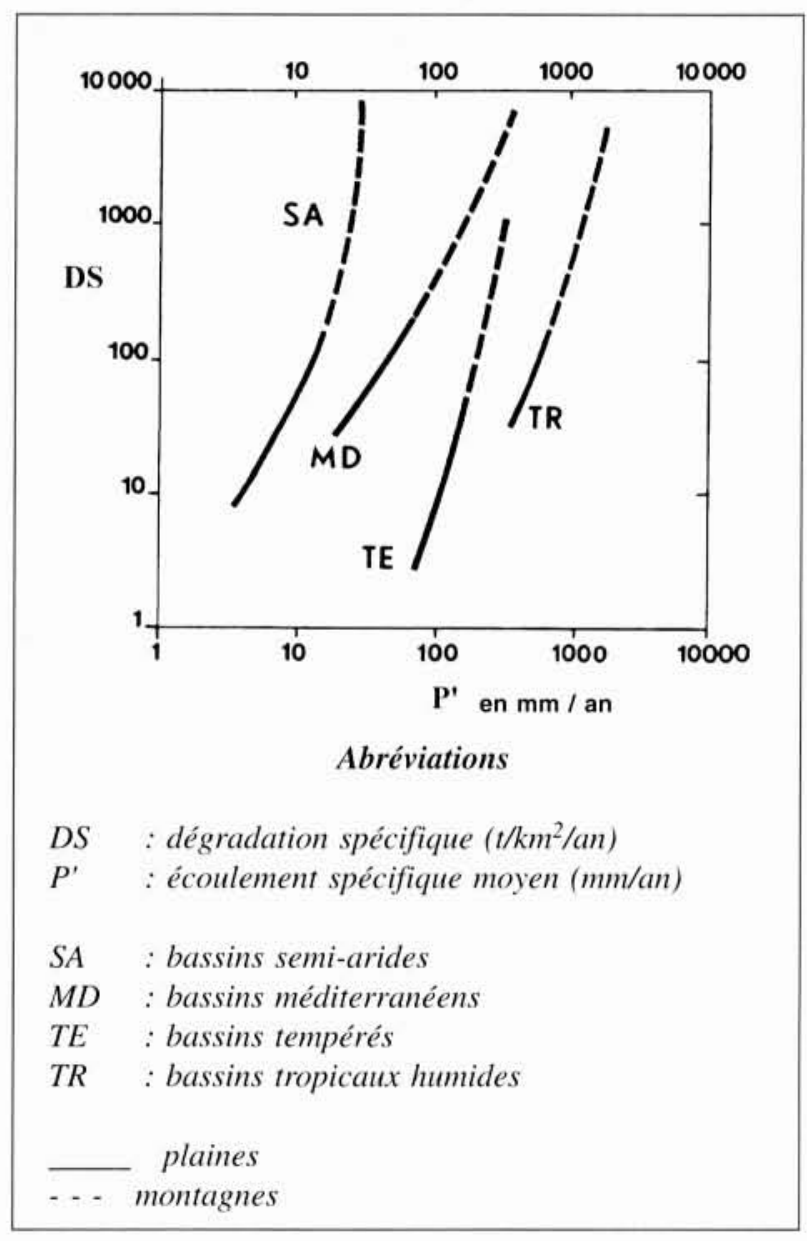

2. Efficacité érosive des bioclimats calculée sur la turbidité des cours d'eau [2]. 
Tableau 2. - Estimations des débits liquides et solides moyens de quelques fleuves classés par ordre décroissant de leur débit solide.

\begin{tabular}{|c|c|c|c|c|c|c|c|c|}
\hline \multirow{3}{*}{ Fleuves } & \multicolumn{2}{|c|}{ Bassin versant } & \multicolumn{5}{|c|}{ Cours inférieur } & \multirow{3}{*}{ Réf. } \\
\hline & \multirow{2}{*}{$\begin{array}{c}\text { Sup. } \\
\left(10^{3} \mathrm{~km}^{2}\right)\end{array}$} & \multirow{2}{*}{$\begin{array}{c}\text { Dégrad. } \\
\text { spécif. } \\
\left(\mathrm{t} / \mathrm{km}^{2} / a n\right)\end{array}$} & \multicolumn{3}{|c|}{ Débit liquide } & \multicolumn{2}{|c|}{ Débit solide } & \\
\hline & & & $\begin{array}{l}Q_{l} \min \\
\left(\mathrm{m}^{3} / \mathrm{s}\right)\end{array}$ & $\begin{array}{c}Q_{l} m o y \\
\left(\mathrm{~m}^{3} / \mathrm{s}\right)\end{array}$ & $\begin{array}{c}Q_{l} \max \\
\left(m^{3} / s\right)\end{array}$ & $\begin{array}{l}C_{s} m o y \\
(m g / l)\end{array}$ & $\begin{array}{c}Q_{s} \\
\left(10^{6} \text { t/an }\right)\end{array}$ & \\
\hline \multicolumn{9}{|l|}{ Gange } \\
\hline Bramahpoutre & 1478 & 1130 & & 31000 & & 1700 & 1670 & $\mathrm{~L}$ \\
\hline $\begin{array}{l}\text { Huang Ho } \\
\text { (Fleuve jaune) }\end{array}$ & 680 & $\begin{array}{c}1330 \\
\left(10000^{\mathrm{a}}\right)\end{array}$ & & 1350 & 25000 & $\begin{array}{c}36900 \\
\left(1.5 \quad 10^{6 \mathrm{a}}\right)\end{array}$ & 1570 & $\mathrm{I}, \mathrm{S}$ \\
\hline Amazone & 5908 & 152 & 50000 & $\begin{array}{c}100000 \\
\text { à } 200000\end{array}$ & & $\begin{array}{c}70 \\
\text { à } 200\end{array}$ & $\begin{array}{c}400 \\
\text { à } 600\end{array}$ & C,I,O,L,P \\
\hline Mississippi & 3265 & 153 & & 18000 & 70000 & 600 & 200 à 344 & $\mathrm{C}, \mathrm{I}, \mathrm{O}, \mathrm{P}$ \\
\hline \multicolumn{9}{|l|}{ Yangzi } \\
\hline \multirow{3}{*}{$\begin{array}{l}\text { Yuchang } \\
\text { Datong } \\
\text { Embouchure }\end{array}$} & 1000 & 518 & & 14300 & & 1180 & 521 & I \\
\hline & 1700 & 280 & & 29200 & & 540 & 478 & I \\
\hline & 1827 & 262 & & 28500 & & 531 & 478 & $\mathrm{P}$ \\
\hline Irrawady & 432 & 660 & & 13500 & & 670 & 285 & $\mathrm{~L}$ \\
\hline Missouri & 1300 & 181 & & 1950 & & 3900 & 240 & I \\
\hline Magdalena & 240 & 920 & & 7500 & & 930 & 220 & $\mathrm{~L}$ \\
\hline Orénoque & 1000 & 210 & & 35000 & & 190 & 210 & $\mathrm{~L}$ \\
\hline Mékong & 800 & 200 & & 14900 & & 340 & 160 & $\mathrm{~L}$ \\
\hline Indus & 1000 & 100 & & 7500 & & 420 & 100 & $\mathrm{~L}$ \\
\hline MacKenzie & 1670 & 60 & & 9600 & & 330 & 100 & $\mathrm{~L}$ \\
\hline \multicolumn{9}{|l|}{ Rhône } \\
\hline \multirow{2}{*}{$\begin{array}{l}\text { Beaucaire } \\
\text { Embouchure }\end{array}$} & 95 & 295 & 400 & 1710 & 10000 & & 28 & $\mathrm{R}, \mathrm{G}$ \\
\hline & 99 & & 400 & 1600 & 13000 & 204 & 5 & $\mathrm{~A}, \mathrm{O}, \mathrm{P}$ \\
\hline Oued & & & 0 & & 130 & $3.10^{5 a}$ & 5 à 20 & M \\
\hline \multirow{3}{*}{$\begin{array}{l}\text { Garonne } \\
\text { Mas d'Agenais } \\
\text { Bec d'Ambès }\end{array}$} & & & & & & & & \\
\hline & 52 & & $<70$ & 630 & 5700 & & & $\mathbf{J}$ \\
\hline & 81 & 250 & & & & & 1 à 3 & $\mathrm{~F}, \mathrm{M}$ \\
\hline Seine & 75 & 17 & $<75$ & 500 & 2500 & & 1 à 2 & $\mathrm{~F}, \mathrm{M}, \mathrm{O}$ \\
\hline Gambie & 42 & & 10 & 150 & & 62 & 0,3 & $\mathrm{P}$ \\
\hline Ebre & 86 & 290 & & 385 & & & 0,2 & E \\
\hline
\end{tabular}

a : maximum local

Références A:[29] - C:[40]-E:G. Gemaehling, 1957 - F:[30] - [9]- I:Institut d'Hydraulique et d'Electricité de Wuhan, 1980 $J:[31]-L:[32]-M:[7]-O:[33]-P:[4]-R:[34]-S:[6]$

\subsection{Impacts sur le bassin versant}

La surface du sol avec son humus, sa pédofaune et sa pédoflore est l'élément essentiel nécessaire pour maintenir ou augmenter la fertilité. Lorsqu'elle est enlevée, il faut des siècles pour qu'elle se reconstitue à partir de la roche-mère. La diminution de la fertilité des terres est la conséquence principale de l'érosion des sols. Duvigneaud [3] estime qu'en un siècle (1870-1970), 10 millions de $\mathrm{km}^{2}$ ont été soustraits aux possibilités culturales du fait de l'érosion. Cette surface représentait $7 \%$ des terres du globe et $14 \%$ des terres actives.

\section{III $\square$ TRANSFERTS DE MASSE EN MILIEUX AQUATIQUES}

\subsection{Le transport solide}

Les milieux aquatiques de surface collectent les particules qui proviennent du lessivage atmosphérique, de l'érosion des sols par les eaux de ruissellement, de l'érosion des berges (pour 20 à $30 \%$ - [4]), et des apports anthropiques directs. Le transfert de l'ensemble de ces particules est couramment appelé "transport solide".

Les différentes régions du globe présentent une grande disparité, géographique et temporelle, des volumes et des types de particules concernés. Le tableau 2 présente les ordres de grandeur de débits liquides $Q_{L}$ et solides $Q_{S}$ de quelques fleuves, sans distinction de l'origine des apports. Les plus grands fleuves présentent des chiffres sur lesquels nous aimerions insister. L'Amazone, par exemple, transporte en une journée moyenne autant de matières en suspension (MES) à son embouchure qu'un oued en quelques jours en période de crue et que la Seine en une année entière. Le Huang Ho, quant à lui, offre un tableau des plus impressionnants. Son débit peut passer de 1350 $\mathrm{m}^{3} / \mathrm{s}$ (débit moyen) à $36000 \mathrm{~m}^{3} / \mathrm{s}$ (en 1843). Il charrie trente fois plus de limon que n'en charriait le Nil avant l'édification du barrage d'Assouan. Dans son lit mineur, le 
Tableau 3. - Transits littoraux de matériaux non cohésifs sous l'effet de la houle (d'après [7]).

\begin{tabular}{|l|c|c|c|}
\hline \multicolumn{1}{|c|}{ Site } & $\begin{array}{c}\text { Transit annuel } \\
\left(10^{3} \mathrm{~m}^{3} / \mathrm{an}\right)\end{array}$ & Transit maximum & Durée correspondante \\
\hline Méditerranée orientale & $50 \mathrm{à} 800$ & $80 \%$ du transit annuel & en moins de 1 mois (tempête) \\
\hline Safi (Maroc) & 250 & $80000 \mathrm{~m}^{3}$ & en 3 jours (tempête) \\
\hline Agadir (Maroc) & 450 & $150000 \mathrm{~m}^{3}$ & en 15 jours (houle moyenne) \\
\cline { 2 - 4 } & à 500 & $85000 \mathrm{~m}^{3}$ & en $7 \mathrm{~h}$ (houle forte $: \mathrm{h}=2,5 \mathrm{~m}, \mathrm{~T}=12 \mathrm{~s})$ \\
\hline Côte du Bénin & 1500 & & - \\
\hline Nord de l'Adour & 300 & \multicolumn{2}{|c|}{-} \\
\hline Manche (Lion-sur-mer) & 50 & \\
\hline
\end{tabular}

Huang Ho coule au-dessus des terres tant son lit s'est exhaussé par ses propres dépôts (montée parfois de $10 \mathrm{~cm}$ par an). En 1855, son embouchure s'est déplacée de $600 \mathrm{~km}$ vers le Nord. Son delta avance actuellement d'environ $1800 \mathrm{~m}$ sur la mer chaque année et gagne une superficie d'environ $32 \mathrm{~km}^{2}$ par an. Enfin, il rompt régulièrement ses digues, ce qui a provoqué notamment 890000 noyades en 1938 [5, 6, 41].

En complément, les transits de matériaux en zone côtière, sous les effets conjugués de la houle, des courants et du vent, peuvent être très importants. En ce qui concerne les matériaux cohésifs, Migniot [7] cite le cas d'un dépôt de 40 millions de $\mathrm{m}^{3}$ de vase d'origine marine, déposé en 6 ans devant l'embouchure de la rivière à Cayenne. Migniot indique également les transits littoraux annuels de matériaux non cohésifs mesurés sur quelques sites et évalue les quantités transportées au cours de courtes périodes critiques ( $\mathrm{cf}$ tableau 3 ).

Les effets de l'activité humaine sur le transport solide, en globalité, sont difficilement quantifiables. Ils peuvent cependant l'être dans quelques cas particuliers.

- La variation des débits solides consécutives aux aménagements des cours d'eau (barrages, endiguements) en est un indicateur. L'Ebre en constitue un exemple extrême. Elle rejette actuellement à son embouchure moins de $1 \%$ de la quantité de sédiments qu'elle transportait à la fin du $\mathrm{XIX}^{\mathrm{e}}$ siècle, avant l'édification de nombreux barrages, soit $0,2 \cdot 10^{6} \mathrm{t} / \mathrm{an}$ au lieu de $25 \cdot 10^{6}$ [8].

- L'impact d'un déboisement sur l'érosion est également évident, si l'on se reporte aux données d'érodabilité en fonction du couvert végétal (fig. 2). Kauark Leité [9] indique que les masses annuellement entraînées par le ruissellement, en milieu tempéré, varient de $100 \mathrm{~kg} / \mathrm{ha}$ en zone forestière à plus de $40 \mathrm{t} / \mathrm{ha}$ lorsque celle-ci a été récemment déboisée, soit 400 fois plus.

- Les activités de dragage constituent également une source de matériaux importante, à la fois pendant les dragages, en mettant artificiellement des particules en suspension, et après les dragages, une partie des matières draguées étant ensuite rejetée. Pour l'année 1979, sur 37 pays recensés par Collins [40], la quantité de matériaux dragués est estimée à 600 millions de tonnes. Elle correspond au débit solide annuel de l'Amazone. Cette même année, le tiers de ces matériaux a été largué en zone océanique et la quasi-totalité du reste en zones côtières ou en milieux aquatiques continentaux.

- L'estimation des apports directs d'origine domestique ou industrielle est parfois possible. Dans les pays développés, ils peuvent représenter une quantité de MES par rapport aux apports "naturels" non négligeable : $80000 \mathrm{t}$ en 1988 sur l'ensemble du bassin Adour-Garonne [10], ce qui représente, suivant les estimations, entre 2,5 et $8 \%$ du débit solide total de la Garonne.

\section{- 3.2 Transferts de polluants autres que les particules solides}

Pour une présentation complète de la nature des principaux polluants autres que les particules solides, en milieu fluvial, ainsi que des origines et causes principales des apports, nous invitons le lecteur à se reporter aux ouvrages d'écotoxicologie, tels ceux de Ramade [11,12]. Les polluants admettent trois formes de présence principales. Ils sont soit dissous, soit fixés sur des particules (en mouvement ou déposées), soit fixés dans le milieu vivant. Ils proviennent essentiellement des rejets industriels, domestiques et urbains, et de la pollution diffuse ou ponctuelle d'origine agricole (cf tableau 4). A la lecture de ce tableau, et en complément d'informations, trois remarques doivent être faites :

1. Rappelons tout d'abord qu'il y a sans cesse transferts de polluants entre phase dissoute, phase particulaire et milieu vivant. Les processus de transferts font l'objet de nombreuses études en écotoxicologie. La connaissance des mouvements des masses d'eau (hydrodynamique) et des transferts associés de particules est à cet effet primordiale. Pour approfondir la description des mécanismes de transport, le lecteur pourra se reporter aux ouvrages généraux $[13,14,15]$ ou à de nombreux travaux traitant plus spécifiquement du transport de sédiments non cohésifs $[16,17,18,19,38]$ ou cohésifs $[20,21,37]$.

2. Concernant les effluents urbains, et à titre d'exemple, le ruissellement sur un bassin urbain imperméabilisé à $30 \%$ provoque un apport de MES compris entre 150 et $1200 \mathrm{~kg} / \mathrm{ha} / \mathrm{an}$, ce qui est du même ordre de grandeur que l'apport des zones rurales peu érodables [22]. En revanche, les effluents urbains sont très chargés en polluants 
Tableau 4. - Principales causes de pollution des eaux autres que les MES en milieu fluvial (classification de Ramade, [12])

\begin{tabular}{|c|c|c|c|c|c|c|}
\hline \multirow[b]{2}{*}{ Type de pollution } & \multirow[b]{2}{*}{ Nature chimique } & \multirow[b]{2}{*}{$\begin{array}{c}\text { Source ou agent } \\
\text { causal }\end{array}$} & \multicolumn{4}{|c|}{ Mode de présence (sur des exemples) } \\
\hline & & & $\begin{array}{c}\text { Paramètres } \\
\text { de pollution } \\
\text { ou polluant, lieu }\end{array}$ & $\begin{array}{l}\text { fraction } \\
\text { particu- } \\
\text { laire }(\%)\end{array}$ & $\begin{array}{l}\text { fraction } \\
\text { dissoute } \\
(\%)\end{array}$ & réf \\
\hline $\begin{array}{l}\text { I- PHYSIQUE } \\
\text { Pollution thermique } \\
\text { Pollution radioactive }\end{array}$ & $\begin{array}{l}\text { Rejets d'eau chaude } \\
\text { Radio-isotopes }\end{array}$ & $\begin{array}{l}\text { Centrales électriques } \\
\text { Installations } \\
\text { nucléaires }\end{array}$ & I, Co, Cs Loire & - & - & G \\
\hline $\begin{array}{l}\text { II- CHIMIQUE } \\
\text { Pollution par les } \\
\text { fertilisants }\end{array}$ & Nitrates, phosphates & Agriculture, lessives & $\begin{array}{l}\text { P Rhône/Léman } \\
\text { Vénoge/Léman }\end{array}$ & $\begin{array}{l}64 \\
36\end{array}$ & $\begin{array}{l}36 \\
64\end{array}$ & $\begin{array}{l}\mathrm{K} \\
\mathrm{K}\end{array}$ \\
\hline $\begin{array}{l}\text { Pollution par les } \\
\text { métaux et métal- } \\
\text { loïdes toxiques }\end{array}$ & $\begin{array}{l}\mathrm{Hg}, \mathrm{Ca}, \mathrm{Pb}, \mathrm{Al}, \mathrm{Zn}, \\
\mathrm{Cu}, \mathrm{Sn}, \mathrm{Cr}, \mathrm{Se}, \mathrm{As}\end{array}$ & $\begin{array}{l}\text { Industrie, agricul- } \\
\text { ture, combustion } \\
\text { (pluies acides) }\end{array}$ & $\begin{array}{ll}\mathrm{Pb}, \mathrm{Zn}, \mathrm{Cu} & \text { Lot infr } \\
\mathrm{Pb}, \mathrm{Zn}, \mathrm{Cu} & \text { Gironde }\end{array}$ & $\begin{array}{c}>80 \\
20\end{array}$ & $\begin{array}{c}<20 \\
80\end{array}$ & $\mathrm{C}$ \\
\hline $\begin{array}{l}\text { Pollution par les } \\
\text { pesticides }\end{array}$ & $\begin{array}{l}\text { Insecticides, herbi- } \\
\text { cides, fongicides }\end{array}$ & $\begin{array}{l}\text { Agriculture, } \\
\text { industrie }\end{array}$ & & & & \\
\hline $\begin{array}{l}\text { Pollution par les } \\
\text { détersifs }\end{array}$ & Agents tensioactifs & $\begin{array}{l}\text { Effluents } \\
\text { domestiques }\end{array}$ & & & & \\
\hline $\begin{array}{l}\text { Pollution par les } \\
\text { hydrocarbures }\end{array}$ & $\begin{array}{l}\text { Pétrole brut } \\
\text { et dérivés }\end{array}$ & $\begin{array}{l}\text { Ind. pétrolière, } \\
\text { transports }\end{array}$ & $\begin{array}{l}\text { Hyd. totaux RPU } \\
\text { HAP }^{2} \mathrm{RPU}^{1}\end{array}$ & $\begin{array}{c}82 \text { à } 99 \\
90\end{array}$ & $\begin{array}{l}1 \text { à } 18 \\
10\end{array}$ & $\begin{array}{l}\mathrm{B} \\
\mathrm{H}\end{array}$ \\
\hline $\begin{array}{l}\text { Pollution par } \\
\text { les composés } \\
\text { organochlorés }\end{array}$ & $\begin{array}{l}\text { PCB, insecticides, } \\
\text { solvants chlorés }\end{array}$ & Industries & $\mathrm{PCB}^{3} \quad \mathrm{RPU}^{1}$ & 93 & 7 & M \\
\hline $\begin{array}{l}\text { Pollution par les } \\
\text { autres composés } \\
\text { organiques de } \\
\text { synthèse }\end{array}$ & $\begin{array}{l}\text { Très nombreuses } \\
\text { molécules }(>70000)\end{array}$ & Industries & & & & \\
\hline $\begin{array}{l}\text { III- } \\
\text { Matières organiques } \\
\text { fermentescibles }\end{array}$ & $\begin{array}{l}\text { Glucides, lipides, } \\
\text { protides }\end{array}$ & $\begin{array}{l}\text { Effluents domes- } \\
\text { tiques, agricoles, } \\
\text { d'industries agro-ali- } \\
\text { mentaires, papeteries }\end{array}$ & $\mathrm{DBO}^{5} \quad \mathrm{RPU}^{1}$ & 77 à 95 & 5 à 23 & B \\
\hline $\begin{array}{l}\text { IV- } \\
\text { Pollution } \\
\text { microbiologique }\end{array}$ & $\begin{array}{l}\text { Bactéries, } \\
\text { virus entériques, } \\
\text { champignons }\end{array}$ & $\begin{array}{l}\text { Effluents urbains, } \\
\text { élevage, agro- } \\
\text { alimentaire }\end{array}$ & & & & \\
\hline
\end{tabular}

': Rejets Pluviaux Urbains sur sites tests; ${ }^{2}$ : Hydrocarbures Aromatiques Polycycliques; ${ }^{3}$ : PolyChloroBiphényls Références B : [21]- C : [25] - G : Girard, $1990-\mathrm{H}:[35]-\mathrm{K}:[8]-\mathrm{M}:[36]$ 
toxiques, lesquels sont pour une très large part véhiculés par les particules, bien plus que sous forme dissoute [23]. 3. Le tableau 4 est qualitatif et ne rend pas compte de l'évolution de la situation, au sujet de laquelle peu de chiffres sont disponibles. L'augmentation des périmètres irrigués (ex : doublement dans le bassin Adour-Garonne dans la période 1982-1992), mais aussi la multiplication des stations d'épuration ou l'augmentation des retraitements industriels ne manquent pas de modifier chaque année la situation.

\section{IV $\square$ IMPACTS DU TRANSPORT PARTICULAIRE}

Le transport particulaire a des répercussions directes et indirectes sur les milieux aquatiques et sur l'activité humaine : directes parce que la modification même des volumes concernés provoque des nuisances, indirectes parce que de leur devenir dépend également celui des polluants divers qui y sont fixés. La fixation par les sédiments de polluants durant leur transfert vers l'aval a des conséquences importantes sur les chaînes alimentaires et sur les cycles biogéochimiques.

\subsection{Impacts directs des particules sur le milieu}

Les problèmes posés en soi par le transport solide concernent essentiellement la morphologie fluviale, aux implications multiples dans le domaine de l'aménagement du territoire, et la modification des équilibres dynamiques des milieux. En zone érodable, l'appauvrissement des terres constitue un effet important du transport solide ( $\mathrm{cf} \S$ 2.2). S'ajoutant au transport " naturel ", les aménagements du cours d'eau, des berges ou du bassin versant et les activités ponctuelles provoquent les déplacements des zones de dépôt et d'érosion et les variations de volume concerné qui entraînent, à moyen ou à court terme, des modifications du lit, des plaines alluviales et des apports côtiers aux conséquences multiples : déchaussement des piles de pont (Loire, Hérault, Garonne), désamorçage des prises d'eau, rabattement des nappes phréatiques, érosion des berges, constitution et migration de bancs de sable, colmatage ou destruction de certaines frayères (Dordogne), érosion des plages (ex : la plage des Sables d'Olonne a perdu au $\mathrm{XX}^{\mathrm{e}}$ siècle $100000 \mathrm{~m}^{3}$ de sable), modification de la turbidité, avec impact sur la photosynthèse et sur l'équilibre des écosystèmes,... Enfin, sur certains bassins et dans le cas de crues paroxysmales, l'envahissement et l'érosion des lits majeurs provoquent, outre les bouleversements écologiques et économiques, des catastrophes humaines et sociales : entraînement de l'habitat provoquant des disparitions humaines, propagation de maladies, généralisation de l'insalubrité et de la précarité.

Du point de vue écologique, les estuaires, les deltas et les retenues artificielles constituent des zones particulièrement sensibles car ce sont des zones privilégiées de dépôt. Une très grande part des polluants chargés en amont s'y dépose. Par exemple, trois millions de $\mathrm{m}^{3}$ supplémentaires de boues s'ajoutent chaque année au fond de la retenue de Serre-Ponçon, sur la Durance [1]. Dans le cas d'un estuaire, il peut s'agir des polluants provenant de l'ensemble d'un bassin versant. Les effets à moyen et long terme des dépôts, dont la nature et les débits évoluent sans cesse ces dernières décennies, sont loin d'être maîtrisés. Ils sont complexes et interdépendants. A ceux-ci, il faut ajouter des conséquences économiques de première importance. Dans le cas d'un barrage, par exemple, l'envasement d'une retenue peut être assez conséquent pour qu'au bout de quelques années l'eau stockée en période de déneigement ou de crues ne soit plus utilisée pour la production hydroélectrique mais pour chasser par les vannes de fond les sédiments accumulés. Dans les réseaux d'assainissement, dont les rejets vont aux milieux naturels, il peut se déposer dans certains collecteurs jusqu'à $0,1 \mathrm{~m}^{3} / \mathrm{m}$ lin./an de solides. Leur extraction coûte actuellement de 300 à $5000 \mathrm{FF}$ par $\mathrm{m}^{3}$. Sachant qu'une ville de 400000 habitants possède environ $500 \mathrm{~km}$ de collecteurs, on conçoit l'ampleur des enjeux écologiques et économiques du " transport solide urbain " [24].

\subsection{Impacts sur les écosystèmes des polluants fixés sur les particules}

Ramade [11] rappelle que toute pollution du milieu vivant, à quelque niveau que ce soit, se propage très vite au niveau de la chaîne alimentaire avec un effet de focalisation de la pollution qui transfère les substances toxiques vers les espèces situées aux niveaux trophiques supérieurs (effets de bio-accumulation et de bio-magnification). L'assimilation par les phytoplancton et zooplancton de polluants initialement dissous ou relargués par les particules se retrouve donc aux niveaux supérieurs de la chaîne alimentaire (crustacés, poissons microphages, poissons prédateurs, oiseaux piscivores,...) avec un impact négatif croissant. En estuaire, les échanges entre phases et milieu vivant sont abondants et limités à un espace parfois restreint, provoquant des modifications, parfois des ruptures, dans les chaînes alimentaires, des transferts, des lésions somatiques, des anomalies physiologiques et des mortalités [25].

A titre dillustrations, citons quelques cas de transferts de polluants et leurs impacts sur le milieu naturel. La présence de particules peut avoir des effets contradictoires sur la flore : ces particules, si elles proviennent d'un bassin agricole, relarguent lorsqu'elles se déposent les composés azotés et phosphatés qui leur étaient fixés et qui favorisent la croissance de certaines algues (biostimulation). Cette dernière, consommatrice d'oxygène, peut affecter la vie benthique. En revanche, un excès de turbidité limite la pénétration du rayonnement solaire, donc la croissance de ces mêmes algues. En ce qui concerne la faune, citons deux exemples. Dans le cours inférieur du Saint-Laurent, les dernières baleines blanches ou bélougas présentent dans leurs tissus des concentrations très élevées de $\mathrm{PCB}$, Benzo-a-pyrène, DDT, $\mathrm{Hg}, \mathrm{Pb}$, provenant essentiellement des effluents urbains et industriels. On a ainsi identifié depuis 1982, par autopsies, des pathologies qui n'avaient jamais été observées sur les baleines : cancers, ulcères perforés de l'estomac, fibrose de la rate [26]. Dans 
l'estuaire de la Gironde, les crevettes concentrent dans leur céphalo-thorax le cadmium provenant des mines fermées et des terrils confinés de Decazeville, sur le cours du Lot, par ruissellement dans les nappes phréatiques (Crié, 1991). Au niveau de l'estuaire, les métaux lourds ne sont présents qu'à $20 \%$ dans la phase particulaire alors qu'ils sont majoritaires sur le Lot inférieur [25]. Il y a transferts entre phases et milieu vivant sur le trajet.

\subsection{Impacts sur les cycles biogéochimiques des polluants fixés sur les particules}

L'étude des cycles des éléments principaux $(\mathrm{C}, \mathrm{N}, \mathrm{O}, \mathrm{P}$, métaux,...) est primordiale pour accroître nos connaissances dans de nombreux domaines consacrés à la dynamique de la planète, et notamment en climatologie. L'évaluation des flux de chaque élément aux interfaces entre les grands systèmes (Terre-Océan-Atmosphère) en est une composante primordiale. La diminution des apports fluviaux en milieux côtiers, consécutifs aux aménagements (barrages, irrigation), permettra-t-elle à l'océan d'accroître l'assimilation de carbone d'origine atmosphérique, contribuant ainsi pour une part, sans doute infime mais qu'il reste à évaluer, à atténuer l'effet de serre induit par l'aug-

\section{Références bibliographiques}

11] Ramade, F. (1981). Ecologie des ressources naturelles, Masson.

[2] Demangeot, J. (1990). Les milieux "naturels" du globe, Masson.

[3] Duvigneaud, P. (1974). La synthèse écologique, Doin Editeurs.

[4] Probst, J.L. et Sigha. N. (1989), Estimation de l'écoulement superficiel et de sa charge en suspension sur quelques grands bassins fluviaux du monde,

C.-R. Ac. Sc. Paris, t. 309, Série II, 357-363.

[5] Favrod, C.H. (1976). Les chinois, Le Livre de Poche.

[6] Scanvic, J.Y. (1987). Le Huang Ho, sa vallée, son delta, approche par télédétection de son aménagement.

[7] Migniot, C. (1977). Action des courants, de la houle et du vent sur les sédiments, La Houille Blanche, 1, 9-47.

[8] Palanques, A. et Guillen, J. (1995). The Ebro delta coastal change : natu ral and human factors, Proc. MedCoast 95, Ozhan E. ed., 897-909.

[9] Kauark Leité, L.A. (1990). Réflexions sur l'utilité des modèles mathématiques dans la gestion de la pollution diffuse d'origine agricole, Thèse de doc torat de I'ENPC

[10] Comité de Bassin Adour Garonne, (1991). Réussir notre futur en AdourGaronne, La politique de l'eau pour l'an 2000, Agence de l'eau AdourGaronne.

[II] Ramade, F. (1977), Ecotoxicologie, Masson.

[12] Ramade, F. (1990). Causes et conséquences écologiques de la pollution de l'eau et des perturbations de son cycle, Le Grand Livre de I'Eau, 367-378, La Manufacture et la Cité des Sciences et de l'Industrie.

[13] Graf, W. (1996). Hydraulique fluviale, T.2 : Ecoulements non permanents et phénomènes de transport, Presses Pol. et Univ. Romandes.

[14] Ramez, P. (1995). Erosion et transport solide en rivière, T.1 : Guide pour la compréhension des phénomènes, Cemagref-Editions.

[15] Bonnefille, R. (1992). Cours d'hydraulique maritime, Masson.

[16] van Rijn. L.C. (1984a). Sediment transport. Part I : Bed load transport. J. Hydr. Eng., ASCE, 110 (10), 1431-1456.

[17] van Rijn, L.C. (1984b). Sediment transport, part II : Suspended load transport, J. Hydr. Eng., ASCE, 110 (11), 1613-1641.

[18] van Rijn, L.C. (1986). Mathematical modeling of suspended sediment in non-uniform flows, J. Hydr. Eng., ASCE, 112, 433-455.

[19] Ouillon, S. et Le Guennec, B. (1996). Modélisation du transport de matières en suspension dans les écoulements $2 \mathrm{D}$ verticaux à surface libre, J. Hydr. Res., AIRH, 34 (2), 219-236

[20] Le Hir, P., Guillaud, J.F., Pommepuy, M., Le Guyader, F. et Salomon, J.C. (1990). Modélisation du transport dissous et particulaire dans l'estuaire de Morlaix, Application au devenir des bactéries entériques, La Houille Blanche, 3/4, 273-278. mentation des rejets de carbone d'origine anthropique ?

\section{$\mathrm{V} \square$ CONCLUSION}

Que conclure, alors que cette introduction appelle mains développements sur tous les sujets abordés? Les conséquences des problèmes liés au transport solide peuvent être d'ordres multiples, écologiques (écosystèmes, couvert végétal, cycles biogéochimiques, climat), humains, sociaux et économiques. Elles sont parfois de première importance. Du seul point de vue écologique, nous avons rappelé que les effets étant cumulatifs, les cycles biologiques et biochimiques sont très sensibles aux modifications en transport solide d'un bassin versant. Nous avons également rappelé que le problème de l'érosion s'inscrit dans le cadre plus général de la diminution et de la dégradation des ressources naturelles dans un monde qui connaît une population croissante. Il constitue de ce fait une des études majeures à conduire en matière de gestion de l'environnement. Concernant les études et recherches sur le sujet, l'interdisciplinarité, s'appuyant sur des méthodes et outils parfois communs, est nécessaire pour améliorer la compréhension de phénomènes interdépendants.

[21] Teisson, C. (1991). Cohesive suspended sediment transpont : feasibility and limitations of numerical modeling, J. Hydr. Res., AIRH, 29 (6), 755-769. [22] Bachoc, A., Mouchel, J.M. et Chebbo, G. (1991). La pollution des rejets pluviaux urbains : son importance, ses caractéristiques, quelques éléments sur ses origines et son interception. Journées Techniques sur les Eaux Pluviales, AGHTM. Agen, Juin 1991.

[23] Chebbo, G. (1992). Solides des rejets pluviaux urbains - Caractérisation et traitabilité, Thèse de doctorat de I'ENPC, CERGRENE. Paris.

[24] Laplace, D. (1991). Dynamique du dépôt en collecteur d'assainissement. Thèse de doctorat $\mathrm{n}^{\circ} 410$ de IINPT, IMFT.

[25] Cabioch, L. (1989). Pollutions en milieu littoral. Le Courrier du CNRS 72, 32-33.

[26] Béland, P. (1991). Opération Bélougas, Sc. et Nat., 10, $42-53$.

[27] Michard, G. (1989). Equilibres chimiques dans les eaux naturelles, Editions Publisud.

[28] Rapinat, M. (1982), L'eau, PUF.

[29] Rivas, J. (1992). Communication personnelle, Agence de l'Eau RhôneMéditerranée-Corse.

[30] Fournier. F. (1960). Climat et érosion, PUF

[31] Junkes, D. (1992). Communication personnelle, Agence de I'Eau AdourGaronne.

[32] Milliman, J.D. et Meade, R.H. (1983). World-wide delivery of river sediment to the oceans, Journal of Geology, 91 (1), 1-21.

[33] Rochefort, M. (1969). Les fleuves, PUF

[34] Ritter, J. (1973). Le Rhône, PUF.

[35] Hermann, R. et Kari, F.G. (1990). Grain size dependant transport of non polar organic trace pollutants ( $\mathrm{PAH}, \mathrm{PCB}$ ) by suspended sediments during urban storm runoff. Proc. 5th Int. Conf. on Urban Storm Drainage, Osaka, Juillet $90,3,499-503$.

[36] Marsalek, J. (1985). Toxic contaminants in urban runoff : a case study. Urban Runoff Pollution, Nato ASI Series, G10, 39-57.

[37] Migniot C. (1987). Manuel sur l'hydrodynamique sédimentaire - Service technique central des Ports maritimes et Voies navigables.

[38] Ramette M. (1981). Guide d'hydraulique fluviale - EDF DER.

[39] Baumgartner, A. et Reichel, E. (1975). The world water balance, Elsevier.

[40] Collins, M. (1989). The behavior of cohesive and non cohesive sediments. Actes Sem. Int. « Aspects environnementaux liés aux activités de dragage $n$, Nantes.

[41] Couderc, J.M. (1991). Erosion et télédétection, Colloque « Terre et environnement ", SCOT Conseil, Toulouse. 